\title{
AVALIAÇÃo DO POTENCIAL DE CONTAMINAÇÃO DE ÁGUAS SUBTERRÂNEAS POR PESTICIDAS: COMPARAÇÃo ENTRE MÉTOdOS DE PREVISÃo DE LIXIVIAÇÃO
}

\author{
CAROLINA LOURENCETTI* \\ CLÁUDIO APARECIDO SPADOTTO** \\ MARY SANTIAGO-SILVA*** \\ MARIA LUCIA RIBEIRO****
}

\begin{abstract}
Este trabalho teve como objetivo comparar alguns modelos descritos na literatura (critérios de screening da EPA, índices GUS e LIX e modelos RF, AF e TLPI) para avaliação do potencial de lixiviação de pesticidas e a possibilidade de contaminação de água subterrânea. Inicialmente, a simbologia e as expressões matemáticas foram organizadas de modo a facilitar a identificação e o entendimento dos fatores considerados na construção de cada modelo. A aplicação e a comparação dos modelos foram realizadas a partir de dados da literatura (propriedades físico-química dos pesticidas, propriedades do solo e condições climáticas) para nove herbicidas (2,4-D, alaclor, atrazina, clomazona, fluazifop-p-butílico, metribuzim, picloram, simazina, trifluralina) registrados e mais usados na cultura de cana-de-açúcar no Brasil. Os resultados do potencial de lixiviação fornecidos pelos métodos não foram concordantes para alguns herbicidas, entretanto, a classificação de potencial de lixiviação apresentouse muito semelhante para todos os produtos. As diferenças encontradas podem ser justificadas pelas singularidades e limitações inerentes à concepção de cada método. Os resultados demonstram que modelos mais simples podem ser úteis para avaliar o potencial de contaminação da água subterrânea em grandes áreas de monocultura, fornecendo subsídios para tomadas de decisões mais rápidas e eficientes pelos órgãos competentes.
\end{abstract}

PALAVRAS-CHAVE: ÁGUAS SUBTERRÂNEAS - CONTAMINAÇÃO; LIXIVIAÇÃO DE PESTICIDAS.

* $\quad$ Mestre em Química, Doutoranda em Química, Instituto de Química, Universidade Estadual Paulista (UNESP), Araraquara, SP (e-mail: carolina@posgrad.iq.unesp.br).

** Doutor em Engenharia Agronômica, Pesquisador da Embrapa Meio Ambiente, Jaguariúna, SP.

*** Professora, Doutora em Química, Instituto de Química, UNESP, Araraquara, SP.

**** Doutora em Química, Professora do Programa de Mestrado em Desenvolvimento Regional e Meio Ambiente, Centro Universitário de Araraquara, SP, Pesquisadora do Instituto de Química, UNESP, Araraquara. 


\section{INTRODUÇÃO}

Atividades agrícolas são conhecidas como fontes de poluição difusa (não-pontual) de águas superficiais e subterrâneas, dentre as quais encontra-se a monocultura da cana-de-açúcar (HIRATA et al., 1993; TSIHRINTZIS, FUENTES e GADIPUDI, 1997; LAABS et al., 2002; MATALLO et al., 2003; GOMES, SPADOTTO e LANCHOTTE 2001). A proteção e conservação da qualidade das águas subterrâneas são partes essenciais de sistemas de produção agrícola sustentáveis. Assim, o impacto por pesticidas na qualidade da água subterrânea tem sido assunto de discussão em todo o mundo, especialmente nas áreas em que a água subterrânea é utilizada para consumo humano (LANCHOTE et al., 2000; WORRAL e KOLPIN, 2004; PARAÍBA et al., 2003; PESSOA et al., 2003). Em alguns casos, menos de $0,1 \%$ da quantidade de pesticidas aplicados alcançam o alvo, enquanto o restante $(99,9 \%)$ tem potencial para se mover para outros compartimentos ambientais como as águas superficiais e subterrâneas (YOUNOS, citado por SABIK, JEANNOT e RONDEAU, 2000). Portanto, a contaminação da água subterrânea por pesticidas pode ocorrer pela percolação da água no solo e através das fraturas dos solos e rochas (FILIZOLA et al., 2002; BRIGANTE et al., 2002).

Programas de monitoramento ambiental, realizados por meio de estudos de campo bem planejados, são considerados por diversos autores como o melhor procedimento de avaliação e de minimização da poluição da água subterrânea (COHEN et al., 1995; ALBANIS et al., 1998; AZEVEDO et al., 2000; FILIZOLA et al., 2002). Esses estudos podem ser realizados analisando-se diretamente a água e/ou o solo, uma vez que o potencial de contaminação da água subterrânea por pesticidas depende da sua mobilidade no solo. Devido ao grande número de princípios ativos utilizados na agricultura, as análises exigem métodos multi-resíduos eficientes e capazes de detectar limites máximos de resíduos estabelecidos pela legislação (LMR) e concentrações consideradas de alerta para a saúde humana (SABIK, JEANNOT e RONDEAU 2000). Estudos de monitoramento apresentam elevado custo e consomem muito tempo devido ao grande número de análises que devem ser realizadas (SPADOTTO et al., 2004). Além disso, quando se trabalha com água subterrânea ocorrem outras limitações na interpretação dos resultados de monitoramento como fator de diluição e repetibilidade na coleta das amostras no mesmo ponto.

Quando não há dados de monitoramento disponíveis, o que ocorre na maioria dos casos, podese recorrer aos métodos de avaliação do potencial de contaminação da água subterrânea por pesticidas para simular a realidade. Esses apresentam diferentes graus de dificuldade, desde a previsão da lixiviação por meio de critérios, índices e modelos matemáticos até a construção de mapas de vulnerabilidade com o auxílio de programas computacionais de sistemas de informações geográficas (SEGURA e LOUREIRO, 1996; COHEN et al., 1995; DIAZ-DIAZ e LOAGUE, 2001; HANTUSH, MARIÑO e ISLAM, 2000), servindo como etapa preliminar dos estudos de monitoramento.

A avaliação da lixiviação de pesticidas no solo pode envolver abordagens diretas ou indiretas. Estimativas diretas incluem a aplicação dos pesticidas no campo (condições são naturais) ou em colunas de solo, podendo-se, portanto, controlar as condições ambientais com análises de amostras do solo em diferentes profundidades (RACKE et al., 1997; OLIVEIRA JÚNIOR, KOSHINEN e FERREIRA, 2001). Já as estimativas indiretas são baseadas na medida de parâmetros que utilizam modelos para avaliação do potencial de lixiviação dos pesticidas no solo (OLIVEIRA JÚNIOR, KOSHINEN e FERREIRA, 2001). Tais modelos são representações físicas, conceituais ou matemáticas da realidade (COHEN et al., 1995).

COHEN et al. (1995), em sua revisão, apresentou o uso de modelos matemáticos para simular o carreamento superficial e a lixiviação de pesticidas para a água subterrânea. Os modelos apresentados são designados com três diferentes propósitos: triagem, regulamentação/exposição e pesquisa. Modelos de triagem (screening/índice) geralmente requerem menor número de dados e são utilizados como advertência para estabelecer prioridades e identificar problemas potenciais. Modelos com o objetivo 
de estabelecer regulamentos podem requerer maior número de dados e são freqüentemente aplicados em avaliações quantitativas de potenciais impactos químicos. Os modelos de pesquisa requerem grande número de dados e apresentam diversos tipos de aplicação.

Técnicas de triagem e índices para avaliação da lixiviação de pesticidas têm sido propostos como estágio inicial para indicar o potencial do impacto dessas substâncias no ambiente, podendo ser aplicados em avaliações de risco de contaminação da água subterrânea por pesticidas (RAO, HORNSBY e JESSUP, 1985; COHEN et al., 1995; PARAÍBA et al., 2003). Essas técnicas são baseadas em propriedades físico-químicas (determinadas de forma empírica ou com solução padrão) com o auxílio de cálculos simples ou de gráficos (COHEN et al., 1995).

Alguns pesquisadores têm tentado estabelecer valores limites para determinada propriedade física ou conjunto de propriedades que, quando excedidos, indicariam que o pesticida apresenta potencial de lixiviação e assim possibilidade de contaminar a água subterrânea. Outros têm proposto modelos analíticos ou numéricos simples, os quais empregam propriedades medidas ou estimadas dos pesticidas e do solo com o objetivo de prever a possibilidade de lixiviação dos mesmos. Alguns dos métodos para previsão da lixiviação de pesticidas apresentados na literatura são: o fator de retardamento RF (Retardation Factor) e o fator de atenuação AF (Attenuation Factor), apresentados por RAO, HORNSBY, JESSUP (1985), o índice GUS (Groundwater Ubiquity Score) proposto por GUSTAFSON (1989), os critérios de screening da EPA (COHEN, 1995), o LIX (Leaching IndeX) proposto por SPADOTTO (2002) e o modelo TLPI ( Temperature Leaching Potenctial Index) conforme mostrado por PARAÍBA e SPADOTTO (2002). Esses métodos podem ser utilizados para selecionar quais substâncias devem merecer maior atenção durante estudos específicos de estimativa da lixiviação de pesticidas no solo e também para obtenção de indicadores ambientais. Alguns dos fatores que influenciam o transporte da molécula de pesticida do solo até a água subterrânea, considerando a persistência e a mobilidade do pesticida no solo, são abordados por esses métodos. Tais fatores envolvem as propriedades físicoquímicas do pesticida (solubilidade, pressão de vapor, coeficiente de partição octanol/água e coeficiente de sorção no solo), as condições ambientais (clima, textura e conteúdo de água do solo), as características da paisagem (topografia e presença de sistemas de drenagem e redes de entrada de escoamento de águas superficiais) e as práticas de manejo (agricultura, seleção da cultura e método de aplicação do pesticida) (ALBANIS et al., 1998; SABIK, JEANNOT e RONDEAU, 2000; MARTÍNEZ et al., 2000). COHEN (1995) alerta para as incertezas inerentes aos modelos de representação do ambiente, pois diferentes locais apresentam variações no tipo de solo, clima, cultura e outros fatores que, em muitos casos, não são considerados pelos modelos.

Os métodos para avaliação do potencial de lixiviação de pesticidas contemplam, isoladamente ou em conjunto, as propriedades dos pesticidas, as condições meteorológicas (ou climáticas), as características topográficas e as práticas de manejo. Há, portanto, métodos muito simples, como os critérios de screening da EPA e os índices GUS e LIX, que consideram somente as propriedades do pesticida (Koc e t1/2), assim como modelos mais simples (como RF, AF, e TLPI) e outros com grande complexidade, que necessitam de conjunto variável de dados e parâmetros.

Embora a comparação entre métodos para previsão da lixiviação e do potencial de contaminação da água subterrânea por pesticidas seja restrita na literatura, trabalhos recentes podem ser citados, como a comparação entre o índice GUS e os critérios da EPA (FERRACINI et al., 2001) e entre os índices GUS e LIX (SPADOTTO, 2002).

Este trabalho teve como objetivo comparar alguns modelos descritos na literatura para avaliação do potencial de lixiviação de pesticidas e a possibilidade de contaminação de água subterrânea, apresentando os fatores considerados e as equações relacionadas na construção dos mesmos. Os herbicidas registrados e mais usados na cultura de cana-de-açúcar no Brasil foram utilizados para efeito de comparação entre os métodos. 


\section{MATERIAL E MÉTODOS}

\subsection{COMPARAÇÃO ENTRE MÉTODOS DE PREVISÃO DA LIXIVIAÇÃO}

Os fatores considerados pelos seis métodos avaliados neste trabalho (critérios da EPA, índices GUS e LIX, e modelos RF, AF e TLPI) foram organizados e apresentados na Tabela 1 com o objetivo de facilitar a identificação dos fatores considerados em cada método.

Os modelos RF, AF e TLPI necessitam de maior número de dados a respeito do solo e do clima, os quais foram identificados em alguns trabalhos com símbolos diferentes, dificultando a comparação entre os modelos (SPADOTTO, FILIZOLA e GOMES, 2001; MATTOS e SILVA, 1999; PARAÍBA et al., 2003). Assim, para facilitar o entendimento das equações foram apresentados (Tabela 1) os símbolos conforme empregados na literatura.

\section{TABELA 1 - FATORES CONSIDERADOS EM CADA MÉTODO ESTUDADO}

\begin{tabular}{|c|c|c|c|c|c|c|}
\hline & $E P A$ & GUS & $L I X$ & $\boldsymbol{R F}$ & $\overline{A F}$ & $\overline{T L P I}$ \\
\hline \multicolumn{7}{|c|}{ Propriedades do pesticida } \\
\hline Koc & $X$ & $X$ & $\mathrm{X}$ & $x$ & $X$ & $X$ \\
\hline$K_{H}$ & $X$ & - & - & $X$ & $\mathrm{X}$ & $X$ \\
\hline$t^{1} 1 / 2$ (solo) & $X$ & $X$ & - & & $\mathrm{X}$ & $X$ \\
\hline t $1 / 2$ (água) & $X$ & - & - & & - & - \\
\hline $\mathbf{S}$ & $X$ & - & - & & - & $X$ \\
\hline$\Delta \mathbf{H}_{\mathrm{v}}$ & - & - & - & & - & $X$ \\
\hline $\mathbf{P v}$ & - & - & - & & - & $X$ \\
\hline$K_{D}$ & - & - & - & & - & $X$ \\
\hline $\mathrm{Ea}$ & - & - & - & & - & $X$ \\
\hline K & - & - & $X$ & & - & $\mathrm{X}$ \\
\hline \multicolumn{7}{|c|}{ Propriedades do solo } \\
\hline FC ou $\theta_{\mathrm{FC}}$ & - & - & - & $X$ & $X$ & $X$ \\
\hline$\rho$ & - & - & - & $X$ & $X$ & $X$ \\
\hline$\delta$ ou na & - & - & - & $X$ & $X$ & $\mathrm{X}$ \\
\hline OC ou foc & - & - & - & $X$ & $X$ & $X$ \\
\hline \multicolumn{7}{|c|}{ Características climáticas e hidrológicas } \\
\hline $\mathbf{T}$ & - & - & - & & - & $\mathrm{X}$ \\
\hline L, d ou z & - & - & - & $X$ & $X$ & $X$ \\
\hline q ou Jw & - & - & - & $X$ & $X$ & $X$ \\
\hline
\end{tabular}

Koc = coeficiente de adsorção à matéria orgânica do solo $\left(\mathrm{m}^{3} \mathrm{~kg}^{-1}\right)\left(\mathrm{mL} \mathrm{g}^{-1}\right.$ para os cálculos de GUS e LIX); $\mathbf{K}_{\mathrm{H}}=$ coeficiente de partição ar-água do pesticida (adimensional); $\mathbf{t}^{1} / 2=$ tempo de meia-vida do pesticida (dia); $\mathbf{S}=$ solubilidade do pesticida em água $\left(\mathrm{g} \mathrm{m}^{-3}\right) ; \Delta \mathbf{H}_{\mathrm{v}}=$ entalpia de volatilização $\left(\mathrm{kJ} \mathrm{mol}^{-1}\right) ; \mathbf{P v}=$ pressão de vapor do pesticida $(\mathrm{Pa}) ; \mathbf{K}_{\mathrm{D}}=$ coeficiente de sorção do pesticida; $\mathbf{E a}=$ energia de ativação da degradação do pesticida $\left(\mathrm{kJ} \mathrm{mol}^{-1}\right) ; \mathbf{k}=$ taxa de degradação do pesticida no solo (dia-1); FC ou $\boldsymbol{\theta}_{\mathrm{FC}}=$ capacidade de campo do solo (fração); $\boldsymbol{\rho}=$ densidade do solo $\left(\mathrm{kg} \mathrm{m}^{-3}\right) ; \boldsymbol{\delta}$ ou $\mathbf{n a}=$ porosidade do solo na capacidade de campo (fração); OC ou $\boldsymbol{f}_{\mathrm{oc}}=$ carbono orgânico do solo (fração); $\mathbf{T}=$ temperatura do solo $(\mathrm{K}) ; \mathrm{L}, \mathbf{d}$ ou $\mathbf{z}$ = profundidade da água subterrânea (ou da profundidade considerada) a uma superfície $\left(\mathrm{m}\right.$ ); $\mathbf{q}$ ou $\mathbf{J}_{\mathrm{w}}=$ recarga líquida da água subterrânea $\left(\mathrm{m} \mathrm{dia}^{-1}\right)$.

As diferentes classificações compiladas da literatura para avaliação dos resultados da aplicação dos métodos estudados são apresentadas na Tabela 2. 


\section{TABELA 2 - CLASSIFICAÇÕES DO POTENCIAL DE LIXIVIAÇÃO DOS PESTICIDAS PARA OS} DIFERENTES MÉTODOS USADOS

\begin{tabular}{|c|c|c|c|}
\hline Modelo & Classificação & Modelo & Classificação \\
\hline $\begin{array}{c}\text { GUS }^{1} \\
<1,8 \\
1,8 \text { a } 2,8 \\
\geq 2,8\end{array}$ & $\begin{array}{c}\text { Potencial de Lixiviação } \\
\text { Nulo } \\
\text { Zona de transição } \\
\text { Potencial de Lixiviação }\end{array}$ & $\begin{array}{c}\mathbf{L I X}^{\mathbf{2}} \\
0 \\
0,0 \text { a } 0,1 \\
\geq 0,1\end{array}$ & $\begin{array}{c}\text { Potencial de lixiviação } \\
\text { Nulo } \\
\text { Zona de transição } \\
\text { Potencial de Lixiviação }\end{array}$ \\
\hline \multicolumn{2}{|c|}{ Critérios EPA ${ }^{3}$} & LTPI $^{4}$ & Potencial de lixiviação \\
\hline $\begin{array}{c}\text { Solubilidade em água } \\
\qquad \mathrm{Koc} \\
\mathrm{K}_{\mathrm{H}} \\
\mathrm{DT}_{50} \text { no solo } \\
\mathrm{DT}_{50} \text { na água }\end{array}$ & $\begin{aligned} & >30 \mathrm{mg} / \mathrm{L} \\
< & 300-500 \mathrm{~mL} / \mathrm{g} \\
< & 10^{-2} \mathrm{~Pa} \mathrm{~m} / \mathrm{mol} \\
> & 14-21 \mathrm{dias} \\
& >175 \text { dias }\end{aligned}$ & $\begin{array}{l}0 \text { a } 24 \\
24 \text { a } 49 \\
49 \text { a } 74 \\
74 \text { a } 89 \\
89 \text { a }+\infty\end{array}$ & $\begin{array}{l}\text { Muito baixo } \\
\text { Baixo } \\
\text { Moderado } \\
\text { Alto } \\
\text { Muito alto }\end{array}$ \\
\hline $\begin{array}{c}\text { Fator de atenuação } \\
(\mathrm{AF})^{5}\end{array}$ & Potencial de lixiviação & $\begin{array}{c}\text { Fator de retardamento } \\
(\mathrm{RF})^{5}\end{array}$ & Potencial de adsorção \\
\hline 0,0 & Nulo & 1,0 & Muito baixo \\
\hline 0,0 a $1.0 \mathrm{E}-04$ & Muito baixo & 1,0 a 2,0 & Baixo \\
\hline $1,0 \mathrm{E}-04$ a $1,0 \mathrm{E}-02$ & Baixo & 2,0 a 3,0 & Médio \\
\hline $1,0 \mathrm{E}-02$ a $1,0 \mathrm{E}-01$ & Médio & 3,0 a 10,0 & Alto \\
\hline 1,0E-01 a 2,50E-01 & $\begin{array}{l}\text { Alto } \\
\text { Muito alto }\end{array}$ & $>10,0$ & Muito alto \\
\hline
\end{tabular}

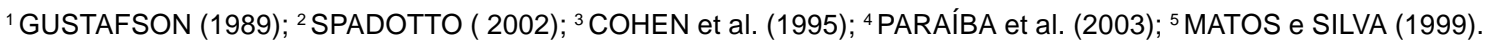

Além dos parâmetros descritos na Tabela 2, COHEN et al. (1995) sugeriram acrescentar aos critérios de screening da EPA as seguintes condições de vulnerabilidade do campo: recarga (precipitação $>25 \mathrm{~cm}$ ano-1), presença de nitrato (altos níveis em água subterrânea são indícios de contaminação por pesticidas) e características do aqüífero (não-confinado e solo poroso sobre o aqüífero). Conforme tais autores, quando todas as características químicas e as condições do meio concordam com os critérios desse modelo, o potencial de contaminação de água subterrânea é alto.

As expressões matemáticas usadas nos índices GUS e LIX e nos modelos RF, AF e TLPI estão reunidas e apresentadas nas equações de 1 a $5 \mathrm{~d}$.

$$
G U S=\log \left(t_{\frac{1}{2}}\right) \times\left(4-\log K_{O C}\right)
$$

Na qual:

$t^{1} 1 / 2=$ meia-vida de degradação do pesticida.

Koc $=$ coeficiente de sorção normalizado ao teor de carbono orgânico do solo.

$$
L I X=\exp \left(-k \times K_{O C}\right)
$$

Na qual:

$\mathrm{k}=$ constante de degradação de primeira ordem do pesticida no solo.

$$
A F=\exp \left(\frac{-0,693 \times L \times R F \times F C)}{q \times t_{\frac{1}{2}}}\right)
$$

Na qual:

$\mathrm{L}$ = profundidade da água subterrânea (ou profundidade considerada) a partir da superfície do solo.

$q$ = recarga líquida da água subterrânea.

$\mathrm{FC}=$ capacidade de campo do solo. 


$$
R F=1+\left(\frac{\rho \times O C \times K_{O C}}{F C}\right)+\left(\frac{\delta \times K_{H}}{F C}\right)
$$

Na qual:

$\rho=$ densidade do solo.

$\mathrm{OC}=$ teor de carbono orgânico do solo.

$\delta=$ porosidade do solo na capacidade de campo.

$\mathrm{K}_{\mathrm{H}}=$ coeficiente de partição ar-água do pesticida.

$T L P I=\frac{1000 \times q}{k(L, t) \times L \times R F(L, h) \times F C}$

$k(L, t)=\left(\frac{\ln (2)}{t_{\frac{1}{2}}}\right) \exp \left(\frac{E a}{R}\left(\frac{1}{T_{r}+273}-\frac{1}{T(L, t)}\right)\right)$

Na qual:

$\mathrm{Ea}=$ energia de ativação da degradação do pesticida.

$\mathrm{R}=$ constante dos gases $\left(8,314 \times 10^{-3} \mathrm{~kJ} \mathrm{~mol}^{-1} \mathrm{~K}^{-1}\right)$.

$\mathrm{Tr}=$ temperatura de referência $\left(20\right.$ ou $\left.25^{\circ} \mathrm{C}\right)$.

$\mathrm{T}=$ temperatura do solo.

$R F(L, t)=1+\frac{\rho \times K_{D}(L, t)}{F C}+\frac{\delta \times H(L, t)}{F C}$

Na qual:

$\mathrm{K}_{\mathrm{D}}=$ coeficiente de sorção do pesticida.

$\mathrm{H}=$ coeficiente de partição do pesticida ar-água.

$$
K_{D}(L, t)=\left(O C \times K_{O C}\right) \exp \left(\frac{\Delta H_{a}}{R}\left(\frac{1}{T r+273}-\frac{1}{T(L, t)}\right)\right)
$$

Na qual:

$\Delta \mathrm{Ha}=$ entalpia de sorção $\left(\mathrm{kJ} \mathrm{mol}^{-1}\right)$.

$H(L, t)=\left(\frac{P m \times P v}{S \times R \times(T r+273)}\right) \exp \left(\frac{\Delta H v}{R}\left(\frac{1}{T r+273}-\frac{1}{T(L, t)}\right)\right)$

Na qual:

$\mathrm{Pm}=$ peso molecular do pesticida $\left(\mathrm{g} \mathrm{mol}^{-1}\right)$.

$\mathrm{PV}=$ pressão de vapor do pesticida.

$\mathrm{S}=$ solubilidade do pesticida em água.

$\Delta \mathrm{H}_{\mathrm{v}}=$ entalpia de volatilização.

Embora os resultados previstos pelo índice GUS (descritos na literatura) concordem na sua maioria com estudos de investigação da presença de pesticida em água subterrânea nos EUA, esse índice prediz valores negativos para moléculas com tempo de meia-vida curto e/ou alto coeficiente de sorção. Tal fato não encontra respaldo no conhecimento básico sobre o processo físico considerado 
(SPADOTTO, 2002). O propósito do uso do LIX, conforme enfatizado por SPADOTTO (2002), não é simular o transporte de pesticidas numa situação de campo, mas sim avaliar o potencial de lixiviação de um composto, comparando a sua lixiviabilidade com a de outra molécula no mesmo cenário ambiental. Tal observação é válida também para os critérios da EPA e o índice GUS.

As aplicações dos modelos RF (Retardation Factor) e AF (Attenuation Factor) demandam levantamento de dados pedológicos, climáticos, hidrológicos, além de propriedades físico-químicas dos pesticidas. Como as propriedades do solo podem ser levantadas por camadas (ou por horizontes), os resultados de RF e AF podem ser também obtidos para cada camada investigada. Os valores podem ser apresentados em rankings comparativos do potencial de lixiviação de cada pesticida nos solos estudados e, conseqüentemente, a possibilidade dos pesticidas atingirem a água subterrânea (MATTOS e SILVA, 1999).

O modelo TLPI (além dos mesmos parâmetros exigidos para o cálculo de RF e AF), por incluir a dependência sobre a temperatura do solo, necessita de dados mais difíceis de serem obtidos como a energia de ativação de degradação, a entalpia de sorção e de volatilização do pesticida. HULSCHER e CORNELISSEN (1996) realçaram a importância da temperatura na cinética e equilíbrio de sorção de compostos orgânicos, apontando a inexistência de consenso quanto ao efeito prático da temperatura no processo de sorção, ou seja, não há relação linear entre o aumento da temperatura e o aumento/ diminuição da sorção.

\subsection{HERBICIDAS ESTUDADOS}

Levantamento efetuado junto à Associação dos Plantadores de Cana-de-Açúcar da região de Araraquara-SP e no comércio agropecuário da mesma cidade indicou que os herbicidas (pré e pósemergentes) são os mais freqüentemente aplicados na cultura da cana-de-açúcar nessa localidade. Portanto, neste trabalho foram avaliados nove herbicidas referenciados por PARAÍBA et al. (2003) que estão na lista de ingredientes ativos das monografias da ANVISA (2004) registrados para uso na cultura de cana-de-açúcar (Tabela 3). O potencial de contaminação de águas superficiais e subterrâneas por esses herbicidas tem sido objeto de pesquisas no Brasil (FILLIZOLA et al., 2002; MATALLO et al., 2003; PESSOA et al., 2003; BRONDI e LANÇAS, 2004) e em outros países (RITTER, 1990; WALLS, SMITH e MANSELL, 1996; BENGTSON, SELIM e RICAUD, 1998; THURMAN, BASTIAN e MOLLHAGEN, 2000; WOOD, GUMBS e HEADLEY, 2002).

É importante observar que os herbicidas cianazina, pendimetalina, sulfentrazona e tebutiuron não foram incluídos na lista de compostos selecionados neste trabalho devido à dificuldade de obtenção de suas propriedades físico-químicas na literatura.

\section{TABELA 3 - PROPRIEDADES DOS HERBICIDAS ESTUDADOS}

\begin{tabular}{|c|c|c|c|c|c|c|c|}
\hline Herbicida & $\begin{array}{c}\mathrm{MM} \\
\left(\mathrm{g} \mathrm{mol}^{-1}\right)\end{array}$ & Pv (Pa) & $\mathrm{S}\left(\mathrm{g} \mathrm{m}^{-3}\right)$ & $\begin{array}{c}\text { Koc } \\
\left(\mathrm{m}^{3} \mathrm{~kg}^{-1}\right)\end{array}$ & t $1 / 2$ (dia) & $\begin{array}{c}\mathbf{k} \\
\left(\mathrm{dia}^{-1}\right)\end{array}$ & $\mathrm{K}_{\mathrm{H}}$ \\
\hline $2,4-D$ & 221 & $1,86 \times 10^{-2}$ & 20031 & $2,00 \times 10^{-2}$ & 10 & 0,0693 & $5,3 \times 10^{-9}$ \\
\hline Alaclor & 269,8 & $2,00 \times 10^{-3}$ & 170,31 & $1,50 \times 10^{-1}$ & 15 & 0,0462 & $1,3 \times 10^{-9}$ \\
\hline Atrazina & 215,7 & $3,85 \times 10^{-5}$ & 33 & $1,00 \times 10^{-1}$ & 77 & 0,0090 & $6,1 \times 10^{-9}$ \\
\hline Clomazona & 239 & $1,92 \times 10^{-3}$ & 1100 & $3,00 \times 10^{-1}$ & 30 & 0,0231 & $1,7 \times 10^{-9}$ \\
\hline $\begin{array}{l}\text { Fluazifop- } p \text { - } \\
\text { butílico }\end{array}$ & 3834 & $333 \times 10^{-5}$ & 11 & 570 & 15 & $0046 ?$ & $44 \times 10^{-9}$ \\
\hline Metribuzim & $\begin{array}{l}214,3 \\
214,4\end{array}$ & $5,80 \times 10^{-5}$ & 1050 & $6,00 \times 10^{-2}$ & 40 & 0,0173 & $4,0 \times 10^{-9}$ \\
\hline Picloram & 241,5 & $8,40 \times 10^{-5}$ & 430 & 5,00 & 30 & 0,0231 & $1,9 \times 10^{-9}$ \\
\hline Simazina & 201,7 & $2,94 \times 10^{-6}$ & 6,2 & $9,50 \times 10^{-2}$ & 60 & 0,0116 & $2,3 \times 10^{-9}$ \\
\hline Trifluralina & 335,3 & $6,10 \times 10^{-3}$ & 0,18 & 8,00 & 60 & 0,0116 & $6,1 \times 10^{-9}$ \\
\hline
\end{tabular}

Fonte dos dados: PARAÍBA et al. (2003) e TOMLIM (1995). MM = massa molar; Pv = pressão de vapor; $\mathrm{S}=$ solubilidade em água; $t^{1} 1 / 2=$ tempo de meia-vida do pesticida no solo; $k=$ taxa de degradação de primeira ordem do pesticida $\left(k=\ln 2 / t^{1} / 2\right)$; $\mathrm{K}_{\mathrm{H}}=$ constante de Henry (adimensional); Koc = coeficiente de sorção normalizado ao carbono orgânico do solo. 


\subsection{APLICAÇÃO DOS MÉTODOS PARA PREVISÃO DA LIXIVIAÇÃO}

A comparação dos métodos utilizados para a avaliação da contaminação da água subterrânea foi efetuada a partir de trabalhos que investigaram o potencial de lixiviação de pesticidas. Os cálculos dos índices GUS e LIX e dos modelos RF e AF para os nove herbicidas estudados foram realizados empregando as equações de 1 a 4 . Os dados necessários para esses cálculos, tais como propriedades físico-química dos pesticidas, propriedades do solo e condições climáticas foram transcritos do trabalho de PARAÍBA et al. (2003) (Tabelas 3 e 4), assim como os resultados do modelo TLPI.

PARAÍBA et al. (2003) aplicaram o modelo TPLI para diferentes camadas $(0$ a $2 \mathrm{~m})$ dos solos Neossolo quartzarênico órtico (TONQ) e Latossolo vermelho distrófico (TDRL), cujas propriedades constam na Tabela 4.

TABELA 4 - PROPRIEDADES DOS SOLOS UTILIZADAS NOS CÁLCULOS*

\begin{tabular}{|c|c|c|c|c|c|c|c|c|}
\hline \multirow{2}{*}{$\begin{array}{c}\text { Camadas } \\
(\mathrm{m})\end{array}$} & \multicolumn{4}{|c|}{$\overline{T O N Q}^{\top}$} & \multicolumn{4}{|c|}{$\overline{T D R L^{2}}$} \\
\hline & $\rho\left(\mathrm{kg} \mathrm{m}^{-3}\right)$ & $\delta(\%)$ & $\begin{array}{l}\mathrm{OC} \\
(\%)\end{array}$ & $\begin{array}{l}\text { FC } \\
\text { (\%) }\end{array}$ & $\rho\left(\mathrm{kg} \mathrm{m}^{-3}\right)$ & $\delta(\%)$ & $\mathrm{OC}(\%)$ & FC (\%) \\
\hline $0,00-0,20$ & 1661,98 & 0,1702 & 0,48 & 16,70 & 1316,1480 & 0,1638 & 1,67 & 31,47 \\
\hline $0,20-0,40$ & 1633,41 & 0,1944 & 0,40 & 15,60 & 1315,8738 & 0,1795 & 1,50 & 32,07 \\
\hline $0,40-0,60$ & 1603,28 & 0,2125 & 0,33 & 15,01 & 1294,1792 & 0,1928 & 1,34 & 32,78 \\
\hline $0,60-0,80$ & 1570,93 & 0,2241 & 0,27 & 15,01 & 1246,6078 & 0,2038 & 1,18 & 33,61 \\
\hline $0,80-1,00$ & 1535,13 & 0,2282 & 0,23 & 15,74 & 1164,6649 & 0,2128 & 1,03 & 34,63 \\
\hline $1,00-1,20$ & 1498,36 & 0,2267 & 0,21 & 16,92 & 1065,3400 & 0,2193 & 0,88 & 35,72 \\
\hline $1,20-1,40$ & 1466,74 & 0,2244 & 0,18 & 17,93 & 990,6894 & 0,2227 & 0,75 & 36,54 \\
\hline $1,40-1,60$ & 1442,73 & 0,2232 & 0,16 & 18,49 & 957,7025 & 0,2226 & 0,65 & 36,95 \\
\hline $1,60-1,80$ & 1425,11 & 0,2221 & 0,14 & 18,74 & 957,8846 & 0,2191 & 0,58 & 37,03 \\
\hline $1,80-2,00$ & 1413,22 & 0,2208 & 0,13 & 18,74 & 986,7794 & 0,2125 & 0,52 & 36,81 \\
\hline
\end{tabular}

\footnotetext{
* Valores apresentados por PARAÍBA et al. (2003).

${ }^{1}$ Neossolo quartzarênico órtico.

2 Latossolo vermelho distrófico.

$\rho=$ densidade do solo; $\delta$ = porosidade do solo na capacidade de campo; OC = carbono orgânico do solo; $F C=$ capacidade de campo do solo.
}

Os valores da recarga líquida da água subterrânea $\left(q\right.$ ou $\mathrm{J}_{\mathrm{w}}$ ), estimados pela técnica de balanço hídrico aproximado, usando dados de precipitação diária, evapotranspiração, irrigação e considerando ainda o grupo hidrológico de cada solo foram: $0,0018 \mathrm{~m} \mathrm{dia}^{-1}$ para o TONQ e 0,0021 $\mathrm{m} \mathrm{dia}^{-1}$ para 0 TDRL (PARAíBA et al., 2003).

Os resultados dos modelos RF, AF e TLPI foram obtidos para cada camada do solo. Como os resultados do TPLI, encontrados por PARAíBA et al. (2003), foram apresentados como valores cumulativos, os valores de RF e AF, calculados nesse trabalho, foram assim também representados.

\section{RESULTADOS DE DISCUSSÃO}

A aplicação dos critérios da EPA, dos índices GUS e LIX e dos modelos RF, AF e TLPI para os nove herbicidas, apresentados na Tabela 3, resultou na elaboração das Tabelas 5 para os critérios e índices e da Tabela 6 para os modelos. 

APLICAÇÃO DOS CRITÉRIOS E ÍNDICES

\begin{tabular}{cccc}
\hline Herbicida & EPA * ${ }^{\mathbf{T}}$ & GUS $^{* 2}$ & LIX $^{*{ }^{3}}$ \\
\hline $2,4-\mathrm{D}$ & $\mathrm{PL}$ & $2,70(\mathrm{ZT})$ & $0,250(\mathrm{PL})$ \\
Alaclor & $\mathrm{PL}$ & $2,14(\mathrm{ZT})$ & $0,001(\mathrm{ZT})$ \\
Atrazina & $\mathrm{PL}$ & $3,77(\mathrm{PL})$ & $0,406(\mathrm{PL})$ \\
Clomazona & $\mathrm{PL}$ & $2,25(\mathrm{ZT})$ & $0,001(\mathrm{ZT})$ \\
Fluazifop-p-butílico & $\mathrm{I}$ & $0,29(\mathrm{~N})$ & $\sim 0(\mathrm{~N})$ \\
Metribuzim & $\mathrm{PL}$ & $3,56(\mathrm{PL})$ & $0,354(\mathrm{PL})$ \\
Picloram & $\mathrm{I}$ & $0,44(\mathrm{~N})$ & $\sim 0(\mathrm{~N})$ \\
Simazina & $\mathrm{I}$ & $3,59(\mathrm{PL})$ & $0,334(\mathrm{PL})$ \\
Trifluralina & $\mathrm{M}$ & $0,17(\mathrm{~N})$ & $\sim 0(\mathrm{~N})$ \\
\hline
\end{tabular}

$\mathrm{PL}=$ potencial de lixiviação; $\mathrm{ZT}=$ zona de transição; $\mathrm{N}=$ nulo; $\mathrm{I}=$ inclonclusivo; $\mathrm{M}=$ mínimo. * Índices calculados a partir dos dados apresentados por PARAÍBA et al. (2003). Critério de classificação: ${ }^{1}$ COHEN et al. (1995); ${ }^{2}$ GUSTAFSON (1989); ${ }^{3}$ SPADOTTO (2002).

TABELA 6 - RESULTADOS E CLASSIFICAÇÃO DO POTENCIAL DE LIXIVIAÇÃO E ADSORÇÃO (RF) COM A APLICAÇÃO DOS MODELOS

\begin{tabular}{|c|c|c|c|c|c|c|}
\hline \multirow[t]{2}{*}{ Herbicida } & \multicolumn{2}{|c|}{$R F^{*}, 1$ (Potencial de adsorçáo) } & \multicolumn{2}{|c|}{$A F * 1$} & \multicolumn{2}{|c|}{$T L P I^{* *, 2}$} \\
\hline & TDRL & TONQ & TONQ & TDRL & TONQ & TDRL \\
\hline $2,4-D$ & $1,46(\mathrm{~B})$ & $1,70(\mathrm{~B})$ & $5,14 \times 10^{-9}(\mathrm{~N})$ & $5,54 \times 10^{-18}(\mathrm{~N})$ & $19(\mathrm{MB})$ & $11(\mathrm{MB})$ \\
\hline Alaclor & $4,43(\mathrm{~A})$ & $6,23(\mathrm{~A})$ & $1,06 \times 10^{-17}(\mathrm{~N})$ & $5,13 \times 10^{-51}(\mathrm{~N})$ & $34(\mathrm{~B})$ & $7(\mathrm{MB})$ \\
\hline Atrazina & $3,28(A)$ & $4,48(\mathrm{~A})$ & $3,56 \times 10^{-3}(\mathrm{~B})$ & $9,24 \times 10^{-8}(\mathrm{~N})$ & $89(\mathrm{~A})$ & $47(\mathrm{~B})$ \\
\hline Clomazona & $7,85(A)$ & $11,45(\mathrm{MA})$ & $8,09 \times 10^{-16}(\mathrm{~N})$ & $3,84 \times 10^{-47}(\mathrm{~N})$ & $18(\mathrm{MB})$ & $9(\mathrm{MB})$ \\
\hline $\begin{array}{c}\text { Fluazifop-p- } \\
\text { butílico }\end{array}$ & 131,23 (MA) & $199,56(\mathrm{MA})$ & $0(\mathrm{~N})$ & $0(\mathrm{~N})$ & $1(\mathrm{MB})$ & $0(\mathrm{MB})$ \\
\hline Metribuzim & $2,37(\mathrm{M})$ & $3,09(A)$ & $4,04 \times 10^{-4}(B)$ & $5,16 \times 10^{-10}(\mathrm{~N})$ & 57 (MD) & $31(\mathrm{~B})$ \\
\hline Picloram & $115,24(\mathrm{MA})$ & $175,18(\mathrm{MA})$ & $9,06 \times 10^{-223}(\mathrm{~N})$ & $0(N)$ & $2(\mathrm{MB})$ & 1 (MB) \\
\hline Simazina & $3,17(\mathrm{~A})$ & $4,31(\mathrm{~A})$ & $9,27 \times 10^{-4}(\mathrm{~B})$ & $2,12 \times 10^{-9}(\mathrm{~N})$ & 71 (MD) & $38(\mathrm{~B})$ \\
\hline Trifluralina & $183,79(\mathrm{MA})$ & $279,69(\mathrm{MA})$ & $8,38 \times 10^{-178}(\mathrm{~N})$ & $0(\mathrm{~N})$ & $2(\mathrm{MB})$ & 1 (MB) \\
\hline
\end{tabular}

$\mathrm{MB}=$ muito baixo; $\mathrm{B}=$ baixo; $\mathrm{M}=$ médio; $\mathrm{MD}=$ moderado; $\mathrm{A}=$ alto; $\mathrm{MA}=$ muito alto; $\mathrm{N}=$ nulo. $\mathrm{TONQ}=$ Neossolo quartzarênico órtico; TDRL = Latossolo vermelho distrófico.

* Modelos calculados a partir dos dados do trabalho de PARAÍBA et al. (2003); ** Valores transcritos do trabalho de PARAÍBA et al. (2003). Critérios de classificação: ${ }^{1}$ MATTOS e SILVA (1999); ${ }^{2}$ PARAÍBA et al. (2003).

Embora os critérios da EPA definam limites para as propriedades físico-químicas dos pesticidas, não há clareza para a obtenção da classificação do potencial de lixiviação dos mesmos. O número das variáveis (propriedades dos pesticidas) que devem obedecer aos valores estipulados para predizer a classificação não é definido. Autores que aplicaram esse critério usaram o termo inconclusivo para princípios ativos que atendem somente a metade das variáveis (FERRACINI et al., 2001; BRITO et al., 2001). Nos critérios da EPA consta o dado de meia-vida dos pesticidas em água, entretanto essa propriedade é pouco citada na literatura, uma vez que depende das características do ambiente. Sendo assim, os valores de meia-vida dos pesticidas em água não foram utilizados neste trabalho para a avaliação do critério da EPA.

O índice GUS, segundo apresentado por GUSTAFSON (1989), propicia a classificação do potencial de lixiviação baseada em faixas numéricas, enquanto SPADOTTO (2002) sugere a classificação (conforme está na Tabela 2) que foi adotada no presente trabalho. Os dados da Tabela 5 mostram que os índices GUS e LIX apresentam classificações de potencial de lixiviação diferentes para o herbicida 2,4-D, devendo-se observar que o índice GUS igual a 2,70 para esse composto estaria próximo do limite superior da zona de transição para potencial lixiviação.

Quando os critérios da EPA e os índices GUS e LIX são comparados, somente foram obtidos resultados semelhantes para atrazina e metribuzim. Esses resultados sugerem que o uso de um 
único método, que considera apenas as propriedades físico-químicas dos pesticidas, pode não ser suficiente para prever o potencial de lixiviação de pesticidas para a água subterrânea. Isso é particularmente verdadeiro para os critérios da EPA.

Ao comparar os modelos RF e AF (Tabela 6), os diferentes valores das propriedades dos dois solos estudados influenciaram os resultados desses métodos e as respectivas classificações para os herbicidas atrazina, clomazona, metribuzim e simazina. Vale salientar que a constante de meia-vida dos pesticidas não é utilizada no cálculo do RF. Por outro lado, o Latossolo vermelho apresenta maior teor de matéria orgânica (expressa como \% de carbono orgânico na Tabela 4) resultando em maior adsorção e, conseqüentemente, menor potencial de lixiviação. A matéria orgânica é citada como um dos principais constituintes do solo responsáveis pela formação de possíveis ligações químicas com moléculas orgânicas, tais como os pesticidas (LANDRAGFT, SILVA e REZENDE, 1998). Os métodos utilizados no presente trabalho empregaram o coeficiente de adsorção normalizado para o teor de carbono orgânico (Koc), assumindo-se assim que a adsorção ocorre exclusivamente à matéria orgânica do solo.

A adsorção e o tempo de meia-vida dos pesticidas apresentam grande influência no fluxo descendente dos pesticidas no solo. Sabe-se que quanto maior a adsorção menor será a mobilidade da molécula, sendo que para as moléculas com valores de coeficiente de adsorção muito elevados a retenção nas camadas superficiais não permite seu deslocamento por lixiviação. Para as moléculas mais móveis, com valores reduzidos do coeficiente de adsorção, o tempo de meia-vida passa a exercer forte influencia na lixiviação. Quanto maior o seu valor mais elevado será o potencial de contaminação de águas subterrâneas, pois maior será o tempo necessário para degradação durante o fluxo descendente do pesticida no solo (MATTOS e SILVA, 1999).

Diferenças foram também observadas para os herbicidas alaclor, atrazina, metribuzim, simazina e trifluralina quando comparados os resultados do modelo TLPI para os dois solos (Tabela 6). TLPI para o solo TONQ resultou em potencial de lixiviação moderado e alto para os herbicidas atrazina, metribuzim e simazina, enquanto que no modelo AF o potencial de lixiviação das mesmas substâncias foi classificado como baixo. O modelo RF apresentou alto potencial de adsorção, indicando baixo potencial de lixiviação para essas moléculas.

Embora os resultados observados nas Tabelas 5 e 6 indiquem diferentes classificações do potencial de lixiviação para alguns herbicidas, quando esses são colocados em ordem decrescente de potencial (Tabela 7) pode-se observar resultados razoavelmente concordantes para os métodos estudados.

\section{TABELA 7 - CLASSIFICAÇÃO EM ORDEM DECRESCENTE DE POTENCIAL DE LIXIVIAÇÃO DOS HERBICIDAS APÓS APLICAÇÃO DOS MÉTODOS ESTUDADOS}

\begin{tabular}{|c|c|c|c|c|c|c|c|c|}
\hline \multirow{2}{*}{ Herbicida } & \multirow{2}{*}{ GUS } & \multirow[t]{2}{*}{ 니X } & \multicolumn{2}{|c|}{$\mathbf{R F}$} & \multicolumn{2}{|c|}{$\mathrm{AF}$} & \multicolumn{2}{|c|}{ TLPI } \\
\hline & & & TONQ & TDRL & TONQ & TDRL & TONQ & TDRL \\
\hline $2,4-D$ & 4 & 4 & 1 & 1 & 4 & 4 & 5 & 4 \\
\hline Alaclor & 6 & $5-6$ & 5 & 5 & 6 & 6 & 4 & 6 \\
\hline Atrazina & 1 & 1 & 4 & 4 & 1 & 1 & 1 & 1 \\
\hline Clomazona & 5 & $5-6$ & 6 & 6 & 5 & 5 & 6 & 5 \\
\hline $\begin{array}{l}\text { Fluazifop-p- } \\
\text { butílico }\end{array}$ & 8 & $7-9$ & 8 & 8 & 9 & $7-9$ & 9 & 9 \\
\hline Metribuzim & 3 & 2 & 2 & 2 & 3 & 3 & 3 & 3 \\
\hline Picloram & 7 & $7-9$ & 7 & 7 & 8 & $7-9$ & $7-8$ & $7-8$ \\
\hline Simazina & 2 & 3 & 3 & 3 & 2 & 2 & 2 & 2 \\
\hline Trifluralina & 9 & $7-9$ & 9 & 9 & 7 & $7-9$ & $7-8$ & $7-8$ \\
\hline
\end{tabular}

TONQ = Neossolo quartzarênico órtico; TDRL = Latossolo vermelho distrófico. 
O modelo RF apresentou a maior discordância na ordem de classificação do potencial de lixiviação dos pesticidas em relação aos outros métodos. Esse é o único que emprega somente a mobilidade do pesticida, não considerando a degradação do mesmo.

PARAÍBA et al. (2003) sugerem que a contaminação de água subterrânea depende das condições hidrológicas e climáticas e das propriedades do solo e dos pesticidas. Além desses parâmetros, a inclusão da temperatura do solo nos modelos de avaliação é importante e essencial para estimar o potencial de lixiviação de herbicidas em solos tropicais (PARAÍBA e SPADOTTO, 2002; PARAÍBA et al., 2003). Tal fato também é enfatizado por RACKE et al. (1997), uma vez que o comportamento de pesticidas em solos tropicais é ainda pouco investigado (RACKE et al., 1997; RACKE, 2003).

A degradação de pesticidas no solo resulta da combinação de eventos químicos e predominantemente biológicos. Como as atividades enzimáticas e microbiologias são afetadas pela temperatura, a mesma exerce grande influencia no tempo de meia-vida dos compostos orgânicos no solo. Assim, WU e NOFZIGER (1999) relataram que modelos de simulação de pesticidas usados para avaliar o risco de contaminação de água subterrânea por pesticidas deveriam incluir o efeito da temperatura na degradação dos mesmos.

A análise comparativa dos resultados da aplicação de índices e modelos torna-se restritiva pela variação dos valores das propriedades físico-químicas dos pesticidas e ausência de dados sobre diversos princípios ativos na literatura (FERRACINI et al., 2001). Além disso, para muitos princípios ativos não há investigações sobre os metabólitos (produtos de degradação) que podem apresentar maior probabilidade de contaminação ambiental e toxicidade menor ou maior que os princípios ativos iniciais (SABIK, JEANNOT e RONDEAU, 2000; GILLIOM, 2001). Outra limitação no uso dos modelos é o enfoque apenas para substâncias individuais. A aplicação agrícola de pesticidas é, com freqüência, realizada utilizando-se misturas, podendo assim acarretar mudança no comportamento da molécula no ambiente. É importante ressaltar que os efeitos combinados (aditivos, sinérgicos ou antagônicos) são ainda pouco estudados (GILLIOM, 2001; FALCONER, 2002).

Devido à complexidade dos processos envolvidos no comportamento dos pesticidas no ambiente pode-se esperar que modelos que incluem maior número de fatores, tais como as propriedades do solo, dos pesticidas e as condições climáticas, resultem em melhor simulação da realidade. Entretanto, há relatos na literatura de que as respostas de sistemas naturais complexos podem, freqüentemente, ser representadas por modelos de baixa ordem e poucos parâmetros, desde que incorporem os componentes dominantes do comportamento ambiental dos pesticidas (DECOURSEY, 1992).

Embora os modelos não reflitam exatamente a realidade são ferramentas valiosas para avaliações simplificadas do comportamento dos pesticidas no ambiente (COHEN et al., 1995). Alguns modelos vêm sendo utilizados juntamente com sistemas de informações geográficas, em escala regional, para avaliar, de forma preliminar, a vulnerabilidade de contaminação da água subterrânea por fontes de poluição não pontuais, como os pesticidas (DIAZ-DIAZ e LOAGUE, 2001; HANTUSH, MARIÑO e ISLAM, 2000).

\section{CONCLUSÃO}

Os métodos que avaliam o potencial de lixiviação de pesticidas para a água subterrânea podem ser utilizados para priorizar quais compostos devem receber maior atenção em estudos mais complexos, como os de monitoramento ambiental. Os resultados de potencial de lixiviação obtidos foram concordantes, exceto para alguns herbicidas empregados no cultivo da cana-de-açúcar. Essas diferenças são justificadas pelas singularidades e limitações inerentes à concepção de cada método. É importante considerar que a ocorrência de determinado pesticida em água subterrânea depende, além do seu potencial de lixiviação, da vulnerabilidade natural do aqüífero e da quantidade do pesticida aplicado. Métodos de previsão da lixiviação, com diferentes graus de complexidade, associados com análise 
espacial e sistema de informações geográficas podem ser úteis para avaliar o potencial de contaminação da água subterrânea em áreas que apresentam elevado consumo de diversos pesticidas, como a monocultura da cana-de-açúcar. O resultado dessa associação pode fornecer subsídios para tomadas de decisões mais rápidas e mais eficientes.

\begin{abstract}
Assessment of groundwater contamination potential by pesticides: comparison among methods of leaching prediction

This work has as objective to compare some methods described in the literature (EPA screening criteria, indices GUS and LIX, and simple models RF, AF and TLPI) for evaluation of pesticide leaching potential and possibilities of groundwater contamination. Initially, the symbols and the mathematical equations were organized in order to facilitate the identification and the understanding of the factors considered during the construction of each model. The application and the comparison of the models were accomplished using literature data (physical and chemical properties of pesticides, soil characteristics, and weather conditions) for nine herbicides (2,4-D, Alachlor, Atrazine, Clomazone, Fluazifop-p-butylic, Metribuzin, Picloram, Simazine, Trifluralin) registered and extensively used in sugar-cane cultures in Brazil. Leaching potential results were not the same for all herbicides; however, the general classification was very similar for the methods studied. Differences found can be explained by singularities and limitations inherent to the conception of each method. The results showed that simpler models can be helpful for assessment of potential of groundwater contamination in extends areas of monoculture, where a large number of pesticides is applied, providing, thus, subsidies for making decisions more quickly and efficiently by the competent authorities.
\end{abstract}

KEY-WORDS: GROUNDWATER - CONTAMINATION; PESTICIDES LEACHING.

\title{
REFERÊNCIAS
}

ALBANIS, T. A.; HELA, D. G.; SAKELLARIDES, T. M.; KONSTANTINOU, I. K. Monitoring of pesticide residues and their metabolites in surface and underground waters of Imathia (N. Greece) by means of solidphase extraction disk and gas chromatography. Journal of Chromatography A, v. 823, p. 59-71, 1998.

2 ANVISA. Agência Nacional de Vigilância Sanitária. Monografias (ingredientes ativos utilizadosherbicidas - na cultura da cana-de-açúcar). Disponível em: <http://www4.anvisa.gov.br/AGROSIA/asp/ rm_pesquisa_ingrediente.asp>. Acesso em: 29 abr. 2004.

3 AZEVEDO, D. A.; LACORTE, S.; VINHAS, T.; VIANA, P.; BARCELÓ, D. Monitoring of priority pesticides and other organic pollutants in river water from Portugal by gas chromatography-mass spectrometry and liquid chromatography-atmospheric pressure chemical ionization mass spectrometry. Journal of Chromatography A, v. 79 , p. $13-26,2000$.

BENGTSON, R. L.; SELIM, H. M.; RICAUD, R. Water quality from sugarcane production on alluvial soils. Transactions of the ASAE, v. 41 , n. 5 , p. 1331-1336, 1998.

BRIGANTE, J.; ESPÍNDOLA, E. L. G.; POVINELLI, J.; ELER, M. N.; SILVA, M. R. C.; DORNFELD, C. B.; NOGUEIRA, A. M. Avaliação ambiental do rio Mogi-Guaçu: resultados de uma pesquisa com abordagem ecossistêmica. São Carlos: Rima Editora, 2002, 60 p.

BRITO, N. M.; AMARANTE Jr., O. P.; ABAKERLI, R.; SANTOS, T. C.; RIBEIRO, M. L. Risco de contaminação de águas por pesticidas aplicados em plantações de eucaliptos e coqueiros: análise preliminar. Pesticidas: revista de ecotoxicologia e meio ambiente, Curitiba, v. 11, p. 93-104, 2001.

BRONDI, S. H. G.; LANÇAS, F. M. HPLC determination of pesticide residues widely employed in sugar-cane cultures in river water samples. Journal of Liquid Chromatography \& Related Technologies, v. 27, n. 1, p. 171-179, 2004

COHEN, S. Z.; WAUCHOPE, R. D.; KLEIN, A. W.; EADSFORTH, C. V.; GRANEY, R. Offsite transport of pesticides in water mathematical models of pesticide leaching and runoff. International Union of Pure and Applied Chemistry, v. 67, n. 12, p. 2109-2148, 1995.

DECOURSEY, D. G. Developing models with more detail: do more algorithms give more truth? Weed Technology, v. 6, p. $709-715,1992$.

DIAZ-DIAZ, R.; LOAGUE, K. Assessing the potential for pesticide leaching for the pine forest areas of Tenerife. Environmental Toxicology and Chemistry, v. 20, n. 9, p. 1958-1967, 2001. 

águas subterrâneas e superficiais da região de Petrolina (PE) e Juazeiro (BA). Pesticidas: revista de ecotoxicologia e meio ambiente, Curitiba, v. 11, p. 1-16, 2001.

FILIZOLA, H. F.; FERRACINI, V. L.; SANS, L. M. A.; GOMES, M. A. F.; FERREIRA, C. J. A. Monitoramento e avaliação do risco de contaminação por pesticidas em água superficial e subterrânea na região de Guaíra. Pesquisa Agropecuária Brasileira, Brasília, v. 37, n. 5, p. 659-667, 2002.

GILLIOM, R. Pesticides in the hydrologic system-what do we know and what is next? Hydrological Processes, v. 15, p. $3197-3201,2001$.

GOMES, M. A.; SPADOTTO, C. A.; LANCHOTTE, V. L. Ocorrência do herbicida tebuthiuron na água subterrânea da microbacia do córrego espraiado, Ribeirão Preto-SP. Pesticidas: revista de ecotoxicologia e meio ambiente, Curitiba, v. 11, p. 65-76, 2001.

GUSTAFSON, D. I.; Groundwater ubiquity score: a simple method for assessing pesticide leachability. Environmental Toxicology and Chemistry, v. 8, p. 339-357, 1989.

HANTUSH, M. M.; MARIÑO, M. A.; ISLAM, M. R. Models for leaching of pesticides in soils and groundwater. Journal of Hydrology, v. 227, p. 66-83, 2000.

HIRATA, R.; RODRIGUES, L; PARAÍBA, L; BUSCHINELLI, C. Groundwater contamination risk from agricultural activity in São Paulo State (Brazil). In: CHILTON. J. (Ed.). Groundwater and agriculture: the inter-relationship. Wallingford: British Geological Survey, 1993. p. 91-93

HULSCHER, ten Th. E. M.; CORNELISSEN, G. Effect of temperature on sorption equilibrium and sorption kinetcs of organic micropollutants - a review. Chemosphere, v. 32, n. 4, p. 609-629, 1996.

LAABS, V.; AMELUNG, W.; PINTO, A.; ZECH, W. Fate of pesticides in tropical soils of Brazil under field conditions. Journal of Environmental Quality, v. 31, p. 256-268, 2002.

LANCHOTE, V. L.; BONATO, P. S ; CERDEIRA, A. L.; SANTOS, N. A. G. CARVALHO, D.; GOMES, M. A. HPLC screening and GC-MS confirmation of triazine herbicides residues in drinking water from sugar cane area in Brazil. Water, Air, and Soil Pollution, v. 118, p. 329-337, 2000.

LANDGRAF, M. D.; SILVA, S. C. da; REZENDE, M. O. O. Mechanism of metribuzin herbicide sorption by humic samples from peat and vermicompost. Analytica Chimica Acta, v. 18711, p. 1-10, 1998.

MARTÍNEZ, R. C.; GONZALO, E. R.; LAESPADA, M. E. F.; ROMÁN, F. J. S. S. Evaluation of surface- and groundwater pollution due to herbicides in agricultural areas of Zamora and Salamanca (Spain). Journal of Chromatography A, v. 869, p. 471-480, 2000.

MATALLO, M. B.; LUCHINI, L. C.; GOMES, M. A. F.; SPADOTTO, C. A.; CERDEIRA, A. L.; MARIN, G. C. Lixiviação dos herbicidas tebutiuron e diuron em colunas de solo. Pesticidas: revista de ecotoxicologia e meio ambiente, Curitiba, v. 13, p. 83-90, 2003

MATTOS, L. M.; SILVA, E. F. Influência das propriedades de solo e de pesticidas no potencial de contaminação de solos e águas subterrâneas. Pesticidas: revista de ecotoxicologia e meio ambiente, Curitiba, v. 9, p. 103-124, 1999.

OLIVEIRA Jr, R. S.; KOSHINEN, W. C.; FERREIRA, F. A. Sorption and leaching potential of herbicides on Brazilian soils. Weed Research, v. 41, p. 97-110, 2001.

PARAÍBA, L.C.; SPADOTTO, C.A. Soil temperature effect in calculating attenuation and retardation factors. Chemosphere, v. 48, p. 905-912, 2002.

PARAÍBA, L. C.; CERDEIRA, A. L.; SILVA, E. F.; MARTINS, J. S.; COUTINHO, H. L. C. Evaluation of soil temperature effect on herbicide leaching potential into groundwater in the Brazilian Cerrado. Chemosphere, v. 53, p. 10871095, 2003.

PESSOA, M. C. P. Y.; GOMES, M. A. F.; NEVES, M. C.; CERDEIRA, A. L.; SOUZA, M. D. Identificação de áreas de exposição ao risco de contaminação de águas subterrâneas pelos herbicidas atrazina, diuron e tebutiuron. Pesticidas: revista de ecotoxicologia e meio Ambiente, Curitiba, v. 13, p.111-122, 2003.

RACKE, K. D. Release of pesticides into the environmental and initial concentrations in soil, water, and plants. International Union of Pure and Applied Chemistry, v. 75, n. 11-12, p. 1905-1916, 2003.

RACKE, K. D.; SKIDMORE, M. W.; HAMILTON, D. J.; UNSWORTH, J. B.; MIYAMOTO, J.; COHEN, S. Z. Pesticides fate and tropical soils. International Union of Pure and Applied Chemistry, v. 69, n. 6, p. 1349-1371, 1997.

RAO, P. S. C.; HORNSBY, A. G.; JESSUP, R. E. Indices for ranking the potential for pesticide contamination of groundwater. Soil and Crop Science Society of Florida, v. 44, p. 1-8, 1985. 
RITTER, W. F. Pesticide contamination of ground water in the United States: a review. Journal Environment Science and Health, Part B, v. 25, n. 1, p. 1-29, 1990.

34 SABIK, H.; JEANNOT, R.; RONDEAU, B. Multiresidue methods using solid-phase extraction techniques for monitoring piority pesticides, including triazines and degradation products, in ground and surface waters. Journal of Chromatography A, v. 885, p. 217-236, 2000.

SEGURA, J. E. S.; LOUREIRO, C. O. Proposta metodológica para a avaliação da probabilidade de contaminação da água subterrânea com praguicidas. In: CONGRESSO BRASILEIRO DE ÁGUAS SUBTERRÂNEAS, 9., 1996, Salvador. Anais... São Paulo: ABAS, 1996. Disponível em: <http://www.abas.org.br/ index.php?PG=publicacoes\&SPG=anais_virtuais>. Acesso em: 19 mar. 2004.

SPADOTTO, C. A. Screening method for assessing pesticide leaching potential. Pesticidas: revista de ecotoxicologia e meio ambiente, Curitiba, v. 12, p. 69-78, 2002.

SPADOTTO, C. A; FILIZOLA, H.; GOMES, M. A. F. Avaliação do potencial de lixiviação de pesticides em latossolo da região de Guaíra. Pesticidas: revista de ecotoxicologia e meio ambiente, Curitiba, v. 11, p. 127-136, 2001.

SPADOTTO, C. A.; GOMES, M. A. F.; LUCHINI, L. C.; ANDRÉA, M. M. Monitoramento do risco ambiental de agrotóxicos: princípios e recomendações. Jaguariúna: Embrapa Meio Ambiente, 2004. 29 p. (Embrapa Meio Ambiente Documentos, 42).

THURMAN, E. M.; BASTIAN, K. C.; MOLLHAGEN, T. Occurrence of cotton herbicides and insecticides in playa lakes of high plains of West Texas. Science of Total Environment, v. 248, p. 189-200, 2000.

TOMLIN, C. (Ed.). The pesticide manual: incorporating the agrochemicals handbook. $10^{\text {th }}$ ed. Bath: Crop Corporation Publications; Royal Chemical Society, 1995. 1150 p.

TSIHRINTZIS, V. A.; FUENTES, H. R.; GADIPUDI, R. K. GIS-Aided modeling of nonpoint source pollution impacts on surface and ground waters. Water Resources Management, v. 11, p. 207-218, 1997.

WALLS, D.; SMITH, P. G.; MANSELL, M. G. Pesticides in groundwater in Britain. International Journal of Environmental Health Research, v. 6, p. 55-62, 1996.

WOOD, B. P.; GUMBS, F.; HEADLEY, J. V. Distribution and occurrence of atrazine, deethylatrazine, and ametryne residues in groundwater of the tropical Island Barbados. Communications in Soil Science and Plant Analysis, v. 33, n. 15-18, p. 3501-3515, 2002.

WORRALL, F.; KOLPIN, D. W. Aquifer vulnerability to pesticide pollution combining soil, land-use and aquifer properties with molecular descriptors. Journal of Hydrology, v. 293, p. 191-204, 2004.

WU, J.; NOFZIGER, D. L. Incorporating temperature effects on pesticide degradation into a management model. Journal of Environment Quality, v. 28, n. 1, p. 92-100, 1999. 